\title{
471974 - THE EFFICACY OF LOW DOSE RECOMBINANT FACTOR VIIA IN THE PROPHYLAXIS OF BLEEDING DURING ORTHOTROPIC LIVER TRANSPLANTATION
}

\author{
Fawzia A. Fetouh, MD $^{2}$, Maged Salah, MD $^{2}$, Khaled Sedeek, MD/PhD ${ }^{1}$ \\ 1. Anesthesiology, Hershey Medical Center, Hershey, PA, USA \\ 2. Anesthesiology, University of Cairo, Cairo, Egypt
}

Introduction: Liver transplantation is the standard form of treatment for patients with end stage liver disease, with the use of blood product as a standard method for transfusion. Recombinant factor VIIa (rVIIa) may help those patients to acquire less amount of transfusion. This will have an impact not only the morbidity and mortality of the recipient and the donor, but also on the economical aspect of this tremendously expensive procedure. We conducted this study to verify the possible beneficial effects of using rVIIa at a lower dose than the standard dosage, which could have an impact on the future use of rVIIa.

Methods: Twenty-four patients scheduled for orthotropic liver transplantation, divided into 2 groups; a control group and an rVIIa group. Both groups received the same anesthetics enlisted in our protocol for liver transplantation. The rVIIa group received a loading dose of $30 \mathrm{ug} / \mathrm{kg}$ of rVIIa following the induction of anesthesia, followed by a maintenance dose of $5 \mathrm{ug} / \mathrm{kg}$ until the end of the dissection phase. Demographic data, coagulation profile [prothrombin time (PT), prothrombin concentration (PC), partial thromboplastin time (PTT), International normalized ratio (INR)], blood loss, transfusion requirements, the duration of the dissection phase, the duration of surgery, hemoglobin concentration $(\mathrm{Hb})$, and platelet count were done immediately after induction and 1, 2, 3 and 6 hours post induction (dissection phase). Finally, a Doppler assessment of the graft vessels was performed subsequent to anastomosis

Results: The rVIIa group had a lower PT in the first two hours of the dissection phase in relation to the baseline and significantly lower than the control group ( $\mathrm{P}=0.0002)$. The INR showed a significant improvement in the rVIIa group during the dissection phase compared to the control group, and during the first two hours compared with the baseline in the rVIIa group ( $\mathrm{P}=0.0002$ ). When compared to the control group, the rVIIa group had a significant increase in the platelet count, in all samples taken during the dissection phase. There was a significant decrease in the intraoperative requirements of packed red blood cells $(\mathrm{P}=0.014)$, platelets $(\mathrm{P}=0.0005)$ and fresh frozen plasma $(\mathrm{P}=0.01)$ in the rVIIa group compared to the control group

Discussion: We conclude that administering low dose of rVIIa would be helpful during liver transplantation surgery. Improvement in the coagulation profile, transfusion requirements, and consequently postoperative morbidity and mortality could be achieved. References: Roberts HR, Monroe DM, White GC. The use of recombinant factor VIIa in the treatment of bleeding disorders. Blood 2004;104:3858-3864. 